\title{
Topical Issue on Highlights of Planetary Science in Italy
}

\author{
Published online: 1 February 2018 - (C) Società Italiana di Fisica / Springer-Verlag 2018
}

Planetary science has an excellent tradition in Italy. After the pioneering investigations by Galileo Galilei, we may recall, among other landmark studies, the discovery of the first asteroid (Ceres) by Giuseppe Piazzi, and the observations of the planet Mars by Giovanni Schiaparelli.

In spite of this remarkable tradition, at the beginning of the Space Age, the Italian community of planetary scientists was small and sparse. It survived only thanks to the enthusiastic initiative of few scientists (in particular, Giuseppe Colombo, an outstanding dynamicist, and a small group of planetary scientists in Rome where Marcello Fulchignoni and Renato Funiciello, at the Department of Geology, started a study of lunar samples).

During the 1970s, following the extraordinary growth of interest in planetary science associated with the launch of a series of interplanetary space probes (among which, Pioneer and Voyager), the Italian planetology community began to grow significantly. In particular, we should mention important dynamical and theoretical studies carried out in Pisa and the studies of asteroids developed in Torino. In the meantime, in Rome, an initial interest in the exploration of the Moon and Mars was soon followed by investigations on the formation of the Solar System; here a highly productive long-term collaboration was started with Victor S. Safronov and his group at the Institute for the Physics of the Earth in Moscow, who first laid the theoretical basis for the accretion theory to explain the formation of the Solar System.

Among the first pioneers in Italian planetary Science, two scientists, no longer among us, deserve a special mention. Angioletta Coradini and Paolo Farinella were both polymaths, both curious and interested in a wide variety of subjects. Angioletta soon became the driver and the leader of a rapidly growing planetary community in Italy, by promoting an active participation in space missions. Paolo was more devoted to theory and he had brilliant intuitions that still provide a reference frame for the planetary science community worldwide.

As a result, the Italian planetary community began to grow, rapidly reaching a strong international reputation. National meetings began to take place in the 1980s and were held regularly in the last twenty years. The 13th Italian Meeting of Planetary Sciences was held on February 2016 in Bormio, a pleasant village in Valtellina and frequent venue for this series of conferences. In fact, the 1st Italian Meeting of Planetary Sciences was held there exactly twenty years earlier. In these twenty years the number of scientists attending the meeting has increased from less than thirty to about one hundred. By now, the relevance of planetary studies is well recognized by the general astrophysical community in Italy.

At present, several Italian teams of planetary scientists, in several institutes throughout the country, are playing a key role in the design of experiments for space missions, and in the analysis of acquired data. The main areas of expertise and scientific activity comprise general and theoretical studies, such as dynamics and collisional processes, the modeling of the formation of jovian planets, spectroscopic composition studies of planetary surfaces and atmospheres and geological evolution of planetary surfaces.

In this general context, the studies of extrasolar systems are generating a revolution in the field. Italian scientists participate in this line of research at the forefront of planetary science. Thus, the motivation and the decision for organizing this timely Focus Point were most natural.

P. Cerroni, E. Dotto, and P. Paolicchi 